Bull. Mater. Sci., Vol. 17, No. 6, November 1994, pp. 607-613. (9) Printed in India.

\title{
History effects in low-field magnetoresistance of BPSSCO polycrystals
}

\author{
A DAS, A BANERJEE and R SRINIVASAN \\ Inter University Consortium for DAE facilities, University Campus, Khandwa Road, \\ Indore 452 001, India
}

\begin{abstract}
Low-field $(H<40 \mathrm{G})$ magnetoresistance measurements have been made on $\mathrm{Bi}_{1.6} \mathrm{~Pb}_{0.4} \mathrm{Sr}_{2} \mathrm{Ca}_{2} \mathrm{Cu}_{3} \mathrm{O}_{10}$ polycrystals at several temperatures between 80 and $105 \mathrm{~K}$. Considerable hysteresis in $p(H)$ is found in a zero-field-cooled sample when the applied field is increased from 0 to a maximum value and then lowered back to 0 at all temperatures. The observation of hysteresis is taken as an evidence for field trapping in the grains. We show that the hysteresis in $p(H)$ occurs for applied fields much lower than that at which $d \rho(H) / d H$ exhibits a discontinuity. In addition, we find that when the applied magnetic field $\left(H_{a}\right)$ is lowered from a maximum field, the effective intergranular field, $H_{\text {eff }}$, becomes zero for $H_{a}>0$, which gives rise to a minimum in $\rho(H)$.
\end{abstract}

Keywords. Magnetoresistance; hysteresis; high- $T_{c}$ superconductor; BPSSCO.

\section{Introduction}

Polycrystalline samples of high-temperature superconductors (HTSC) are generally recognized as granular superconductors. They consist of superconducting grains

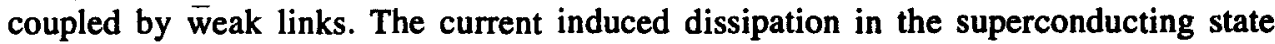
is governed by the presence of two types of current, viz. the intergrain current and the intragrain current. The low value of bulk $J_{c}$ measured in these systems and its reduction by about two orders of magnitude in low fields $(H<100 \mathrm{Oe})$ has been described by a model describing Josephson type of coupling between the grains (Peterson and Ekin 1988).

It is observed that a zero-field-cooled (ZFC) sample in applied field exhibits strong hysteretic effects in $J_{c}$ (Evetts and Glowacki 1988), AC susceptibility (Levy et al 1993), magnetoresistance (Pradhan A K, unpublished), and higher harmonics in magnetization (Shailendra Kumar et al 1992). The hysteresis in these is attributed to the field in the intergranular region, $H_{\text {eff }}$, which is determined by the grain magnetization and therefore depends on the history. It is of importance to note that at a given temperature, hysteretic effects are observable only when the maximum applied field, $H_{m}$, is such that the field is trapped in the grains.

Lopez et al (1992) have made magnetoresistance measurements on a polycrystalline $\mathrm{GdBa}_{2} \mathrm{Cu}_{3} \mathrm{O}_{7}$ sample to obtain the $H_{c / g}(T)$. They identify $H_{c l g}$ with the field at which a discontinuity is obtained in the plot $d R\left(H_{a}\right) / d H_{a}$, in ZFC sample. The argument following this result is that as we increase the field from 0 , in a $\mathrm{ZFC}$ sample, for $H_{a}<H_{c l g}$, the field lines are concentrated in the grain boundaries. Therefore, $R\left(H_{a}\right)$ has strongest field dependence here and when $H_{a}>H_{c l g}$ the intensity of the induction field in the junction is reduced as a result of which a drop in rate of increase in magnetoresistance is observed, and therefore the discontinuity in $d R\left(H_{a}\right) / d H_{a}$. 
We report here low-field magnetoresistance measurements on a polycrystalline $\mathrm{Bi}_{1.6} \mathrm{~Pb}_{0.4} \mathrm{Sr}_{2} \mathrm{Ca}_{2} \mathrm{Cu}_{3} \mathrm{O}_{10}$ sample at various temperatures and show that hysteresis in $R(H)$ exists at fields far below the peak in $d R\left(H_{a}\right) / d H_{a}$. This implies that flux penetrates into the grains at a field much lower than is evident from the suggestion of Lopez et al (1992).

\section{Experimental}

Samples in the form of pellets of nominal composition $\mathrm{Bi}_{1 \cdot 6} \mathrm{~Pb}_{0.4} \mathrm{Sr}_{2} \mathrm{Ca}_{2} \mathrm{Cu}_{3} \mathrm{O}_{10}$ were prepared in the standard ceramic route. For resistance measurements the samples used were of dimension $10 \times 1.1 \times 1.49 \mathrm{~mm}$. Four probe contacts were made by Indium solder and the contact resistance was $<5 \Omega$. A constant AC current of $1 . \mathrm{mA}$ at $23.1 \mathrm{~Hz}$ was passed through the sample. The voltage was sensed by a lock-in amplifier (SRS 530) with a low noise transformer (EG and G PAR 1900) at the input stage. The temperature was measured by a $100 \Omega \mathrm{Pt}$. resistance driven by a $1 \mathrm{~mA}$ dc current from a constant current source (Keithley 224) and the voltage sensed by a DMM (Keithley 196). Temperature was maintained constant within $\pm 0.05 \mathrm{~K}$ at each temperature.

\section{Results and discussion}

The resistivity at room temperature of the sample is $2.54 \mathrm{~m} \Omega \mathrm{cm}$. Figure 1 shows the variation of resistivity, $\rho$ as a function of temperature, $T$, between 80 and 200 $\mathrm{K}$, for $I=1 \mathrm{~mA}$. The variation is linear in $T$ above $130 \mathrm{~K}$. Significant departure from linearity occurs at $T_{c}(\mathrm{On}) \simeq 120 \mathrm{~K}$. Between 113 and $105 \mathrm{~K}$ the rate of decrease in $\rho$ is fairly sharp. Below $105 \mathrm{~K}$ the fall is gradual, i.e. it tails off to finally achieve a $T_{c}$ (zero) at $98 \mathrm{~K}$. Similar tails in $\rho(T, H=0)$ have been observed

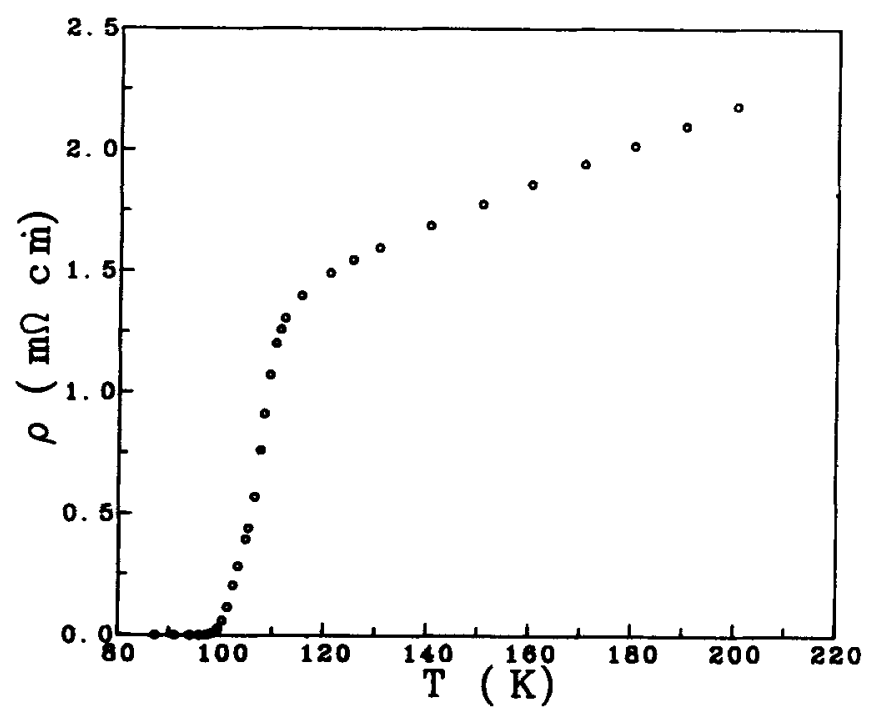

Figare 1. Resistivity ( $\rho)$ as a function of temperature $(T)$. 
in other BSCCO granular materials and is likely a result of weak intergranular coupling. The fall in $\rho$ between 113 and $105 \mathrm{~K}$ is associated with the onset of superconductivity within the grains. Between 105 and $98 \mathrm{~K}$ the decrease in $\rho$ is due to phase incoherence between the grains, and below $98 \mathrm{~K}$ phase coherence sets in. There appears a distinct change in $\rho(T)$ at $105 \mathrm{~K}$ corresponding to $\rho \approx$ $458 \mu \Omega \mathrm{cm}$, i.e. $\rho(105 \mathrm{~K})<\rho(0 \mathrm{~K})$ (extrapolated) $\simeq 1.19 \mathrm{~m} \Omega \mathrm{cm}$. The value of $\rho \geq 458 \mu \Omega \mathrm{cm}$ is identified with the $\rho$ of decoupled grains.

In figures 2-7 the variation of $\rho$ as a function of applied dc magnetic field, $H_{a}$, for temperature $T=81 \cdot 5,85,90,95,100$, and $105 \mathrm{~K}$, respectively are shown. The samples are zero-field-cooled at each temperature, and then $\rho$ is measured in the field increasing $\left(H^{\uparrow}\right)$ mode and in the field decreasing $\left(H_{\downarrow}\right)$ mode from a maximum field, $H_{m}=38 \mathrm{G}$. Pronounced hysteresis is observed between $H^{\uparrow}$ and $H_{\downarrow}$ at all measured temperatures. The value of magnetoresistivity $\Delta \rho(H)(=\rho(H)-\rho(0))$ at any given field increases as the temperature is increased up to $105 \mathrm{~K}$. At $110 \mathrm{~K} \Delta \rho(H) \simeq 0$.

At $T=81.5$ (figure 2) magnetoresistance is observed beyond a field $H_{1}^{\uparrow} \simeq 12 \mathrm{G}$. In $H^{\uparrow}$ mode the nature of the curve is such that the derivative $d R\left(H_{a}\right) / d H_{a}$ always increases and no discontinuity is observed. When the field is lowered from $H_{m}=$ $38 \mathrm{G}$, it is observed that $\rho\left(H_{\downarrow}\right)<\rho\left(H^{\uparrow}\right)$. It is also found that in $H_{\downarrow}$ mode $H_{1 \downarrow} \simeq 15$ $\mathrm{G}$ and for $H_{a}<12 \mathrm{G}, \rho\left(H_{\downarrow}\right)=\rho\left(H^{\uparrow}\right)=0$. For $T=85 \mathrm{~K}$ (figure 3 ) the behaviour of $\rho(H)$ is similar to that at $T=81.5 \mathrm{~K}$ except for $H_{1}^{\uparrow}=H_{1 \downarrow}=9 \mathrm{G}$.

At $T=90 \mathrm{~K}$ (figure 4) the behaviour of $\rho(H)$ departs from the lower temperatures ones. Here $H_{1}^{\uparrow}=3.5 \mathrm{G}$ beyond which $\rho$ increases rapidly and above $H_{2}^{\uparrow}=20 \mathrm{G}$ the rate of rise in $\rho(H)$ decreases, i.e. $d R\left(H_{a}\right) / d H_{a}$ undergoes a discontinuity at $H_{2}$. The resistivity at $H_{2}$ corresponds to $36 \mu \Omega \mathrm{cm}$. On lowering the field from $H_{m}$, $\rho\left(H_{\downarrow}\right)<\rho\left(H^{\uparrow}\right)$ till $H_{3}$, below which $\rho\left(H_{\downarrow}\right)>\rho\left(H^{\uparrow}\right)$. In addition $\rho\left(H_{\downarrow}\right) \neq 0$ when $H_{a}$ is lowered to 0 . This may be termed as the remanent magnetoresistivity analogous to remanent magnetization.

At $T=95 \mathrm{~K}$ (figure 5), $H_{1}^{\uparrow} \simeq 2 \mathrm{G}$, the field $H_{2}^{\uparrow}$ occurs at $6 \mathrm{G}$. Above $H_{2}^{\uparrow}$ the rate of increase falls rapidly and field dependence appears to reach a saturation. On lowering $H_{a}$ from $H_{m}, H_{3}$ is found to shift to higher field i.e. $7 \mathrm{G}$. For $H_{a}<H_{3}$ a minimum in $\rho(H)$ is observed for $H_{a} \simeq 2$ below which the resistance increases on lowering the field. The remanent resistivity increases to $46 \mu \Omega \mathrm{cm}$.

For $T \geq 100 \mathrm{~K}$ (figures 6 and 7 ), $H_{1}=0, H_{2}=0$, i.e. the region below $36 \mu \Omega \mathrm{cm}$ is absent. The resistivity rises very rapidly at small fields and appears to saturate for $H_{a}>10 \mathrm{G}$. On decreasing the field from $H_{m}$ very small hysteresis is observed at $100 \mathrm{~K}$. The hysteresis is nearly absent in the case of $T=105 \mathrm{~K}$. On decreasing $H_{a}$ to 0 , however, remanent magnetoresistivity is observed.

There are two characteristic break points, fields $H_{1}$ and $H_{2}$. The magnetic field causes a shift in the phase difference along the tunnel barrier between the grains thereby reducing $J_{c}, H_{1}$ is the field at which transport current density, $J^{t}$, attains the critical current density, $J_{c}^{t}$ of the weak links. Therefore, the temperature dependence of field $H_{1}(T)$ arises from the temperature dependence of $J_{c}(T)$. The change in the functional dependence of $\rho(H)$ at $H_{2}$ may be for the following reasons (Gerber et al 1982), either (i) at $\mathrm{H}_{2}$ almost all the Josephson junctions are suppressed and the field $H_{2}$ corresponds to effective critical field of the assembly of junctions, $H_{c J}$. Therefore the field dependence of $\rho(H)$ below $H_{2}$ corresponds to that of network of Josephson junctions. Above $\mathrm{H}_{2}$ since the weak links are absent the rate of increase in magnetoresistivity with field sharply reduces. However, 


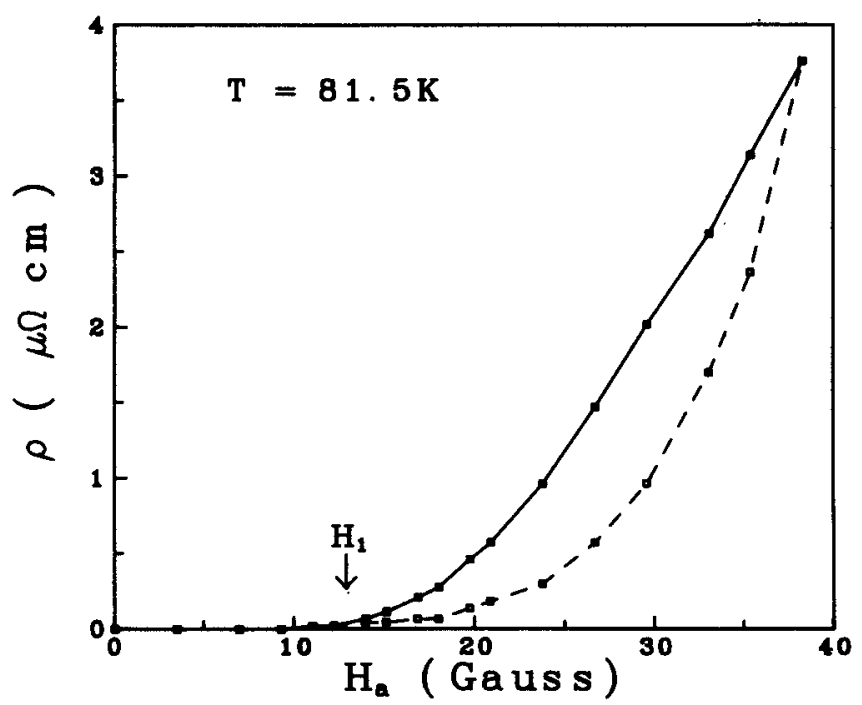

Figure 2. For caption, sec p. 612.

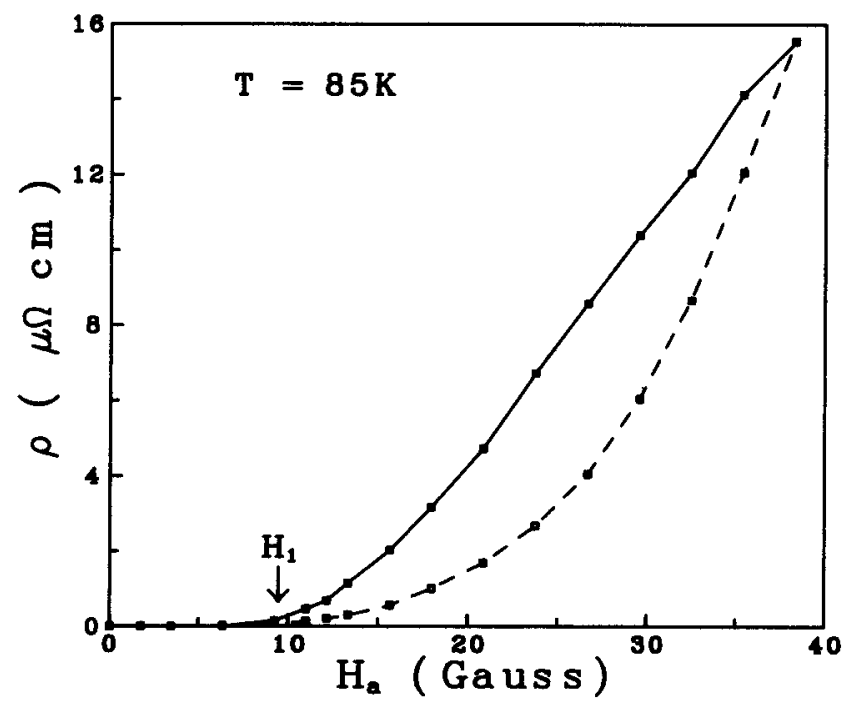

Figure 3. For caption, see p. 612.

an appropriate power law fitting of the form $\rho\left(H_{a}\right) \propto H_{a}^{n}$, is improper to make as in the ZFC case here as the intergranular field is not only hysteretic, but is also affected by the field compression effects, or (ii) $\mathrm{H}_{2}$ corresponds to the field at which the $H_{\text {eff }}\left(H_{2}\right) \geq H_{c l_{s}}$ as also suggested by Lopez et al (1992). The additional flux will now be distributed evenly in the volume of the sample. This would decrease the flux density in the junctions and therefore, the sample resistance will increase slowly for $\mathrm{H}_{a}>\mathrm{H}_{2}$. 


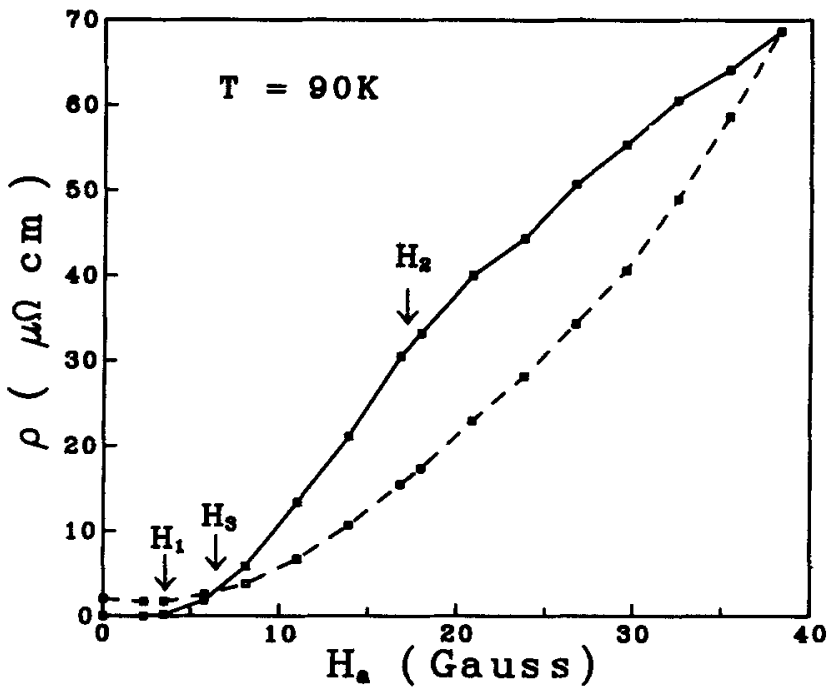

Figure 4. For caption, see p. 612.

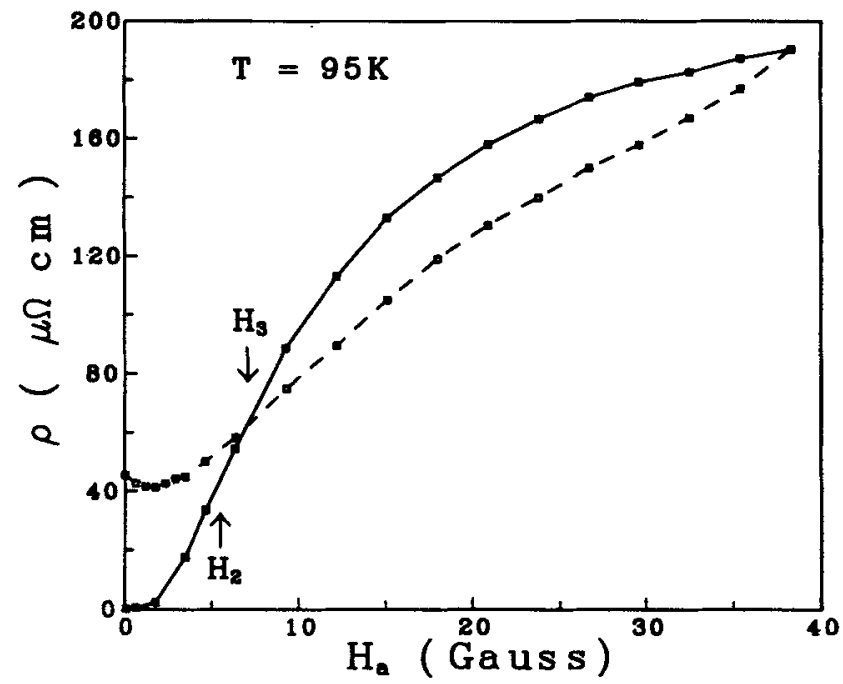

Figure 5. For caption, see p. 612.

We find for $T \leq 85 \mathrm{~K}, H_{2}>H_{m}=38 \mathrm{G}$. But when the field is reduced from $H_{m}$ hysteresis is observed. Hysteresis is a signature of $H_{\text {eff }}\left(H_{m}\right)>H_{c l g}$. Therefore, here $H_{c l g}<H_{2}$. Pradhan A $\mathrm{K}$ (unpublished) from second harmonic and magnetoresistance measurements shows that $H_{c l g}<H_{2}$. Therefore, the second possibility is incorrect. We identify $\mathrm{H}_{2}$ with the field at which majority of the weak links are destroyed. Since the junctions have a distribution of critical fields the transition from weak link dominated $\rho(H)$ to region where $\rho(H)$ arises from flux creep process in the grains is not sharp. The two regions are overlapping. 


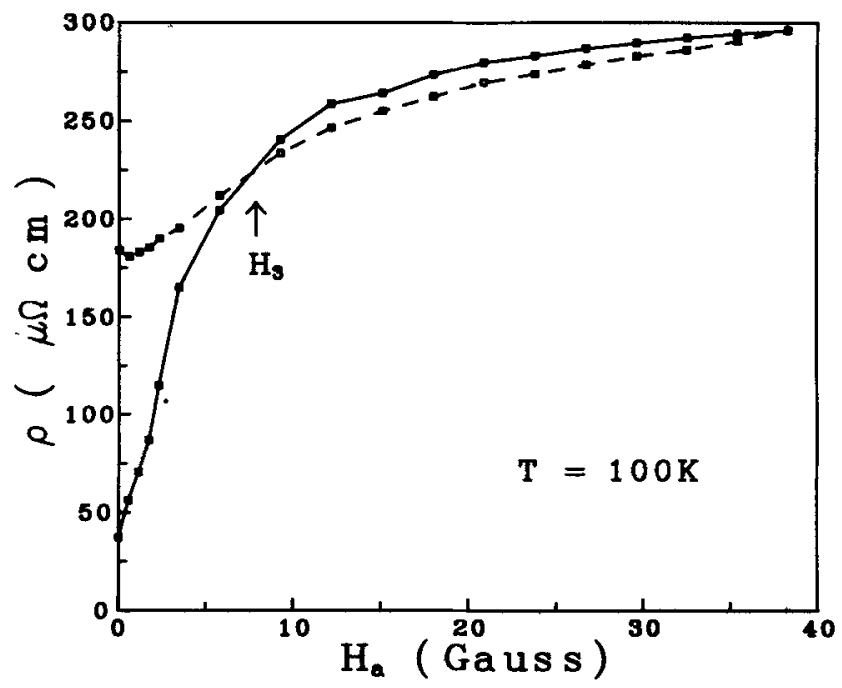

Fïure o.

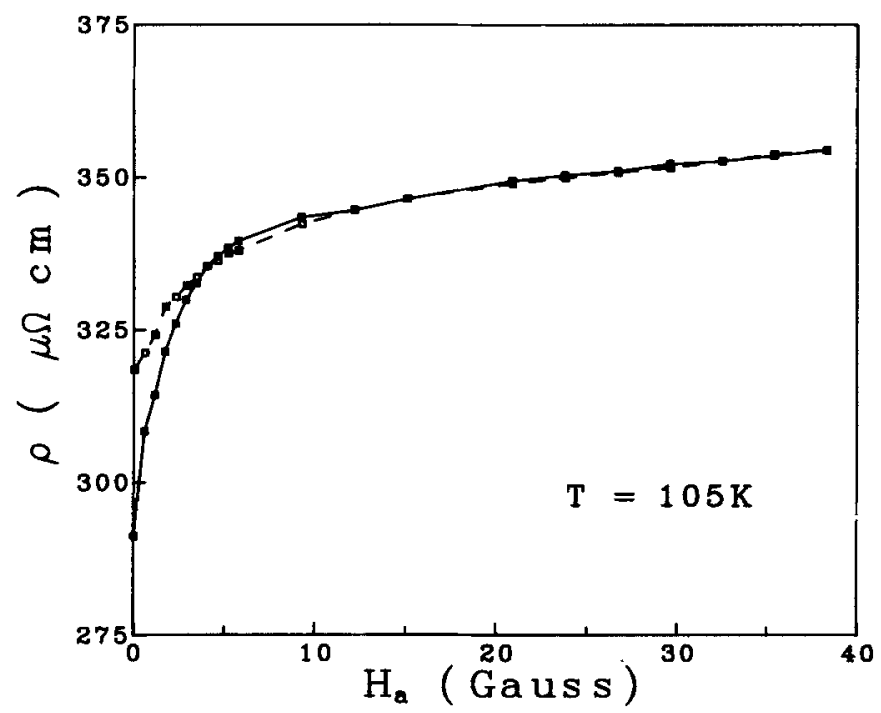

Figures 2-7. Resistivily $(\rho)$ an a function of $\mathcal{D C}$ magnetic field $\left(H_{a}\right)$ at temperatures 81-5, $85,90,95,100,105 \mathrm{~K}$, respectively. The continuous line indicates the field increasing mode $\left(H^{\uparrow}\right)$ and the dashed line indicate the field decreasing mode $(H \downarrow)$.

On reducing the field from $H_{m}, \rho\left(H_{\downarrow}\right)<\rho\left(H^{\uparrow}\right)$. This is understood to be due to vectorial cancellation of $H_{a}$ and the trapped field in the grains which creates a field in a direction opposite to $H_{a}$. Therefore, $H_{\text {eff }}\left(H_{\downarrow}, \mathrm{ZFC}\right)<H_{\text {eff }}\left(H^{\uparrow}, \mathrm{ZFC}\right)$ (Shailendra Kumar et al 1992). At $T=105 \mathrm{~K}$ (figure 7) on reducing the field from $H_{m}$ the hysteresis is nearly absent. This is because as $T$ approaches $T_{c}$ the pinning potential reduces and therefore less trapping in the grains occur which therefore, 
reduces the hysteresis. On lowering $H_{a}$ further, at a field, $H_{\text {comp }}$, the field due to the trapped flux in the grains is exactly compensated by $H_{a}$, i.e. $H_{\text {eff }} \simeq 0$ (Das et al 1994). At $H_{\text {comp }}, J_{c}$ exhibits a maximum (Evetts and Glowacki 1988) and even harmonics a minimum (Roy et al 1992; Das et al 1994). For $H_{a}<H_{\text {comp }}, H_{\text {eff }}$ reverses its sign (Das et al 1994). Since $J_{c} \propto 1 /|H|$ for $H_{a}<H_{\text {comp }}, J_{c}$ decreases. Experimentally it has been observed by other workers that, $H_{\text {comp }}$ increases and then saturates as $H_{m}$ is increased. Furthermore, the peak of $J_{c}$ broadens and its height decreases as we increase $H_{m}$. Both these observations are explained by the model of Müller and Mathews (1993) which takes into account the magnetization as well as the material parameters, viz. the grain boundary length and the probability distribution of the demagnetization factor. We have kept $H_{m}$ constant and increased the temperature. At a fixed temperature raising $H_{m}$ is equivalent to raising $H_{m}$ relative to $H_{c l g}$. But keeping $H_{m}$ fixed, raising temperature lowers $H_{c I g}$ and therefore has the same effect. Therefore, as the temperature is raised the peak value of $J_{c}\left(H_{\downarrow}\right)$ decreases which leads to the observation of non zero remanent magnetoresistance as the temperature increases. Our observation of $H_{\text {comp }}>0$ for BSCCO system is in contradiction with the observation of Muné et al (1994) who find from $J_{c}(H)$ measurements no evidence of $H_{\text {comp }}$ at $77 \mathrm{~K}$. The absence of $H_{\text {comp }}$ is explained on the basis of brick wall model of Bulaevski et al (1992).

\section{Conclusions}

From the present study of $\rho(H, T)$ we find that at all temperatures between 81.5 and $105 \mathrm{~K}$ hysteresis exist between field increasing and decreasing modes. Existence of hysteresis is taken as an indication of $H_{\text {eff }}\left(H_{m}\right) \geq H_{c l g}$. The field at which a discontinuity in $d \rho\left(H_{a}\right) / d H_{a}$ appears is identified with the critical field of the Josephson junction network, $H_{c J}$. We also find that like in YBCO systems in these systems too $H_{\text {comp }}>0$.

\section{Acknowledgement}

We thank Mr $\mathrm{R} \mathrm{V} \mathrm{Krishnan} \mathrm{for} \mathrm{his} \mathrm{help} \mathrm{during} \mathrm{the} \mathrm{various} \mathrm{stages} \mathrm{of} \mathrm{the} \mathrm{work.}$

\section{References}

Bulaevski L N, Clem J R, Glazmạn L I and Malezomoff A P 1992 Phys. Rev. B45 2545

Das A, Bajpai A, Banerjee A and Srinivasan R 1994 Pramana-J. Phys. 43211

Evetts J E and Glowacki B A 1988 Cryogenics 28641

Gerber A, Grenet Th, Cyrot M, and Beille J 1992 Phys. Rev. B45 5099

Levy P, Ferrari H, Bekeris V and Acha C 1993 Physica C214 111

Lopez D, de la Cruz F, Shastry P, Leyarovska N and Matacolta F C 1992 Phys. Rev. B46 11160

Muiller K-H and Mathews D N 1993 Physica C206 275

Muné P, Altshuler E, Musa, J, Garner S and Riere R 1994 Physica C226 12

Peterson R L and Ekin J L 1988 Phys. Rev. B37 9848

Roy S B, Shailendra Kumar, Chaddah P, Ram Prasad and Soni N C 1992 Physica $C 198383$

Shailendra Kumar, Roy S B, Chaddah P, Ram Prasad and Soni N C 1992 Physica C191 450 